\title{
LA FUNCIÓN ARTÍSTICA EN HENRY JAMES A TRAVÉS DE “THE MIDDLE YEARS", “THE DEATH OF THE LION", “THE LESSON OF THE MASTER” Y "THE FIGURE IN THE CARPET"'.
}

\author{
Francisco José López Quintana. Universidad de Almería
}

\begin{abstract}
La pretensión central de este artículo consiste en el análisis de la concepción estética de Henry James a través de cuatro de sus relatos más significativos: "The Middle Years", "The Death of the Lion", "The Lesson of the Master" y "The Figure in the Carpet”. Al final del mismo se pondrá de relieve la analogía existente entre las ideas del escritor americano con las de Stéphane Mallarmé, figura señera del movimiento Simbolista francés, y con las del filósofo alemán Friedrich Nietzsche.
\end{abstract}

\section{INTRODUCCIÓN}

Los relatos titulados "The Middle Years", "The Death of the Lion", "The Lesson of the Master" y "The Figure in the Carpet" giran en torno a la relación del escritor y su obra con el mundo circundante en general, y con el lector en particular ${ }^{2}$. Sin embargo, cada uno de ellos presenta un grado diferente de análisis de esta cuestión. Mientras en "The Middle Years" vemos cómo es el lector quien se convierte en el soporte y justificación ideológica del autor que no consigue vencer su frustración por no haber alcanzado su ideal de perfección, en "The Lesson of the Master", Henry James identifica la vocación artística con una especie de sacrificio monástico, alejado de toda vida social y familiar. "The Death of the Lion" avanza en esa línea, planteando el problema de la fama como obstáculo para el logro de esa perfección, que en último extremo debe conducir a que la obra literaria sea capaz de transmitir un mensaje transcendental, cuya comprensión queda reservada tan sólo para una élite selecta, según se evidencia en "The Figure in the Carpet".

A continuación, intentaremos subrayar los aspectos más relevantes de la concepción estética de Henry James a través de cada uno de los relatos mencionados, de tal forma que nos permitan posteriormente extraer unas conclusiones que contribuyan a una mejor comprensión de las ideas de Henry James en el ámbito referido.

\footnotetext{
${ }^{1}$ Las citas proceden de Henry James, 1986.

${ }^{2}$ En realidad, nos encontramos con un auténtico "conflicto entre el arte y el mundo que sufre el escritor al tratar de desarrollar su papel público" (Álvarez Calleja, 1994: 417).
} 


\section{LOS RELATOS}

A) "The Middle Years":

Aquí, el tema del escritor y su función se tiñe de un carácter filosófico que se manifiesta desde el comienzo. En efecto, la noción de tiempo ocupa un lugar preferencial en el planteamiento argumental de Henry James a través de la dicotomía vejez / juventud ${ }^{4}$, que se desarrolla por medio de una serie secundaria de ideas como es la oposición entre enfermedad ${ }^{5}$ y placer $^{6}$. El resultado de esta antítesis dialéctica se refleja en una concepción pesimista acerca del devenir existencial: la expresión "the abyss of human illusion" (235) encierra el fatalismo vital que impregna todo el relato desde principio a fin.

Pero si analizamos el proceso evolutivo del discurso filosófico, vemos cómo traduce la frustración personal del protagonista, un escritor llamado Dencombe ${ }^{7}$, que se verá ayudado por un lector admirador suyo, el Doctor Hugh ${ }^{8}$. De hecho, gracias a él, Dencombe realiza una reflexión introspectiva por medio de la cual se da cuenta de que su desánimo no se debe a una sensación de temor hacia la muerte, ni de un apego a la vida ${ }^{9}$, sino de algo más relacionado con sus aspiraciones profesionales: "What he dreaded was the idea that his reputation should stand on the unfinished" (247). El Doctor Hugh es un incondicional de Dencombe, y con la veneración que manifiesta hacia é ${ }^{10}{ }^{10}$ trata de hacerle ver el beneficio realizado con su labor literaria ${ }^{11}$. En este sentido, es bastante explícita la expresión que define al Doctor Hugh: "servant of his altar"12 (251). Así, haciéndole sentir importante, el médico intenta que logre vencer esa sensación de perdición que le invade ${ }^{13}$, y que no es otra cosa que un miedo cerval a no poder desarrollar todo su potencial artístico como acabamos de poner de relieve anteriormente. Porque los fantasmas ya mencionados de la enfermedad y la vejez son los que le producen su frustración existencial, al evitar que pueda proseguir con su actividad creativa ${ }^{14}$.

Dencombe, por su parte, al final del relato se esforzará denodadamente para que el Doctor Hugh asegure su situación económica ${ }^{15}$, poniendo todos los medios para que su amigo no abandone a la Condesa -su paciente y protectora-, quien -estando a punto de morir- podría dejarle parte de su herencia. Sin embargo, fracasa en su intento precisamente porque la elección del Doctor Hugh es terminante: opta por su amigo y pone todo su celo para que se sienta debidamente valorado por la sociedad. Por eso, deja de preocuparse de la salud de la Condesa y centra todo su celo en la salvación metafísica de Dencombe. Vuelve junto a su ídolo para mostrarle un artículo literario donde se le hace el justo reconocimiento que merece su obra: "it was an acclamation, a reparation, a critical attempt to place the author in the niche he had fairly

\footnotetext{
3 "Age" (235).

4 "Young experience" (236).

5 "His illness had made him feel his age" (235).

6 " (...) but taking for granted there could be no complete renewal of the pleasure (...)" (236).

7 "He needed help too much" (247).

8 "I want to do something for you" (247).

9 "He wasn't afraid of suffering, of death, wasn't even in love with life" (247).

10 "The young man held his hand, hanging over him, and poor Dencombe, weakly aware of this living pressure, simply lay there and accepted his devotion" (247).

11 "You have done a tremendous lot for me" (247).

${ }^{12}$ Este calificativo también aparece en "The Death of the Lion".

13 "He was lost, he was lost -he was lost if he couldn't be saved" (251).

14 "Illness and age rose before him like spectres with pitiless eyes: how was he to bribe such fates to give him the second chance?" (248).

15 "Poor Dencombe, (...) who only desired that everything should indeed be 'saved"” (255).
} 
won" (256). Sin embargo, la resistencia de Dencombe es persistente: “Ah no -but they would have been true of what I could have done"' (256), por lo que se entabla un forcejeo dialéctico entre ambos personajes, donde el Doctor Hugh trata por todos los medios de convencer a Dencombe de que su misión está cumplida, frente a lo que su interlocutor ofrece continuas objeciones:

'Why it has come to pass -it has come to pass! The second chance has been the public's -the chance to find the point of view, to pick up the pearl!'

'Oh the pearl!' poor Dencombe uneasily sighed. A smile as cold as winter sunset flickered on his drawn lips as he added: 'The pearl is the unwritten -the pearl is the unalloyed, the rest, the lost!' (256)

A la vez, se produce la confirmación del sacrificio que el Doctor Hugh ha realizado, al ratificar su elección por el arte (Dencombe) frente al dinero (la Condesa):

'The Countess is dead', said Doctor Hugh. 'I knew that in a particular contingency she wouldn't resist. I went to her grave'.

Dencombe's eyes opened wider. 'She left you "something handsome"?'

The young man gave a laugh almost too light for a chamber of woe. 'Never a penny.

She roundly cursed me'.

'Cursed you?' Dencombe wailed.

'For giving her up. I gave her up for you. I had to choose', his companion explained.

'You chose to let a fortune go?'

'I chose to accept, whatever they might be, the consequences of my infatuation', smiled Doctor Hugh. Then as a larger pleasantry: 'The fortune be hanged! It's your own fault if I can't get your things out of my head.' (257)

Esta elección de su amigo es la que provoca en Dencombe cierto efecto, haciendo que se sienta satisfecho en su fuero más íntimo y contribuyendo a mejorar su estado de ánimo:

a response so absolute, such a glimpse of a definite result and such a sense of credit, worked together in his mind and, producing a strange commotion, slowly altered and transfigured his despair. The sense of cold submersion left him -he seemed to float without an effort. (257)

No obstante, Dencombe dejará bien claro que no le basta con el hecho de que alguien se haya encontrado atraído con su obra ${ }^{16}$. En realidad, su aspiración es un ideal imposible, conseguir una segunda oportunidad con la que alcanzar la perfección tan anhelada:

'a second chance -that's the delusion. There never was to be but one. We work in the dark -we do what we can- we give what we have. Our doubt is our passion and our passion is our task. The rest is the madness of art'. (258)

El último intercambio de palabras de nuestros personajes es bastante revelador, no tanto por lo que se dice, sino fundamentalmente por lo que está implícitamente sobreentendido:

16 "It is glory -to have been tested, to have had our little quality and cast our little spell. The thing is to have made somebody care. You happen to be crazy of course, but that doesn't affect the law" (258). 
'Frustration's only life', said Doctor Hugh.

'Yes, it's what passes'. Poor Dencombe was barely audible, but he marked with the words the virtual end of his first and only chance. (258)

En efecto, vemos que aquí se ponen de relieve dos ideas básicas y, a la vez, íntimamente relacionadas entre sí: ciertamente, de lo que Dencombe está hablando es de lo efímero tanto de la vida como de su obra, puesto que su carrera se ha visto frustrada por no haber conseguido ese ideal de perfección con el que hubiera podido transcender el tiempo para alcanzar la inmortalidad.

B) "The Lesson of the Master":

En esta historia, Henry James plantea la incompatibilidad de la vida social en general, y más específicamente de la familiar, con la vocación artística entendida en su grado más elevado.

El artista es caracterizado por Paul Overt -un joven escritor admirador de la obra de un autor consagrado, Mr. St. George- a partir de sus peculiaridades físicas y su atuendo:

he had almost always recognised the artist and the man of letters by his personal 'type', the mould of his face, the character of his head, the expression of his figure and even the indications of his dress. ${ }^{17}(123-4)$

Overt detesta la explicación racional de la obra de $\operatorname{arte}^{18} \mathrm{y}$, además, considera que la vida del artista es pobre en comparación con una vida de acción, frente a su lectora Miss Fancourt, para quien el arte está ligado con una vida intensa:

'Well, after all, why try to be an artist?' the young man pursued. 'It's so poor -so poor!'

'I don't know what you what you mean', said Miss Fancourt, who looked grave.

'I mean as compared with being a person of action -as living your works.'

'But what's art but an intense life -if it be real?' (130)

Tras esta introducción, Henry James encauza el principal debate del argumento en torno al ideal artístico, como destaca Mr. St. George al revelar a Paul Overt que sus últimos libros distan bastante de la perfección que él hubiera deseado ${ }^{19}$. Hecho este ya observado por su admirador $^{20}$, quien, a su vez, pone de relieve la misma sensación de superficialidad (138). E incluso se menciona la idea de fracaso por parte de Miss Fancourt: "he thinks he's a failure" (153), frente a la potencialidad que encerraba, según indica Paul Overt: "with his gifts it ought to have been high" (153). En este tema, parecen haber influido ciertas desavenencias existentes entre Mr. St. George y su esposa ${ }^{21}$, que han debido afectar a su función de escritor, según se desprende de algunos detalles como la quema de uno de los libros del famoso novelista llevado

\footnotetext{
17 No obstante, establece una excepción en el caso de Inglaterra, donde la figura del escritor parece despersonalizarse, careciendo de cualquier rasgo definitorio: "In England this identification was as little as possible a matter" (124).

18 "For to his own ear the explanation of a work of art sounded fatuous" (129).

19 "They're not what they should be. He told me he didn't esteem them" (131).

20 "Some of his later books seem to me of a queerness!" (131).

21 "'Do you suppose he understands his wife?"” (133).
} 
a cabo por su mujer (134). En todo caso, estos supuestos problemas familiares ${ }^{22}$ están encaminados a llamar la atención sobre el hecho obvio e indiscutible de que Mr. St. George no ha logrado exhibir toda su extraordinaria capacidad artística en sus últimas publicaciones ${ }^{23}$.

Igualmente, Mr. St. George expresa una sensación de decrepitud artística, que va pareja a su admiración por su discípulo Paul Overt. De este modo, da comienzo la contraposición entre el ocaso de un escritor y la ascensión de otro $^{24}$. Detengámonos ahora en los factores que han intervenido en ese declive literario:

- Se produce una identificación entre "old age" y "worship of false gods", incluyendo en este último grupo al dinero, los hijos y la esposa:

'(...) Don't become in your old age what I have in mine -the depressing, the deplorable illustration of the worship of false gods!'

(...) "The idols of the market; money and luxury and "the world"; placing one's children and dressing one's wife; everything that drives one to the short and easy way. And the vile things they make one do!' (141-2)

- Por otra parte, está la excesiva popularidad, la cual ejerce una influencia negativa sobre el escritor $^{25}$, como refleja su obligación de asistir a gran número de acontecimientos sociales ${ }^{26}$.

El discurso alcanza su clímax con la identificación de la obra de arte con la perfección ${ }^{27}$. Los otros no importan, ni el número, lo que importa es la "decent perfection" para un número reducido de personas (164). O incluso para la propia conciencia (165): “A certain perfection's possible and desirable" (167). Y frente a ese ideal, Mr. St. George desvela el proceso que lleva a la degradación del artista: el problema pecuniario ante el que se ha visto obligado a doblegarse ${ }^{28}$, no es más que el resultado de la vida familiar ${ }^{29}$, cuyo último responsable parece ser el interés crematístico de la mujer en general, y de la suya en particular:

Their idea is that you shall do a great lot and get a great lot of money. Their great nobleness and virtue, their exemplary conscientiousness as British females, is in keeping you up to that. (...) Aren't you the father of their innocent babes, and will you withhold from them their natural sustenance? (168)

De esta forma, el sexo femenino se convierte en el becerro de oro ${ }^{30}$ que no puede tomar parte en el sacrificio exigido al artista, porque no puede entenderlo (171), y además, no se preocupa por la perfección ${ }^{31}$.

\footnotetext{
22 "“Do you think he is unhappy at home?"” (153).

23 "He might have been one of the greatest" (154). En realidad, se trata de la misma idea que perseguía de forma obsesiva a Dencombe, el protagonista de "The Middle Years".

24 "But you must be better, you really must keep it up. I haven't of course. It's very difficult" (140).

25 "“Too many things -too many things!"” (150).

26 "They send him invitations" (149).

27 "The perfection to which for the moment they agreed to confine their speculations was that of the valid, the exemplary work of art" (155).

28 "Mercenary muse whom I led to the altar of literature" (165).

${ }^{29}$ Reflejada por medio de los conceptos "marriage", "wife" y "children" (168).

30 "They're the idol and the altar and the flames" (171).

31 "He said (...) she didn't care for perfection" (154).
} 
Éstos son, pues, los puntos referenciales que permiten el desarrollo expositivo de la lección (141) que Mr. St. George pretende dar su discípulo, ofreciéndose a ayudar a Paul Overt para que resista a todas las tentaciones de las que puede ser objeto y lograr la salvación ${ }^{32}$.

El propósito último se convierte en "the real thing" (166), identificado con la sensación de perfección: "the sense of having done the best" (167). Y aquí entra en juego el concepto de "success" que reúne dos rasgos principales:

a) Frente a la connotación de intranscendencia atribuida al reconocimiento público o fama, la idea de "success" adquiere un sentido más profundo: la capacidad de toda obra consumada para producir un efecto determinado en sus lectores ${ }^{33}$.

b) Y por otro lado, la perfección de la que habla debe conseguirse con sacrificio, a diferencia de lo que ocurre con Mr. St. George, quien a pesar de tener satisfechas todas sus necesidades materiales ${ }^{34}$-o más bien debido a ello-, siente un vacío transcendental ${ }^{35}$. El camino para alcanzar esa perfección ha de pasar, pues, por un desprendimiento de todas las posesiones y ataduras materiales (170), haciendo una especie de voto de pobreza ${ }^{36}$ que permita al escritor mantener en todo momento su independencia ${ }^{37}$. Sólo de esta manera el artista deja de ser un hombre corriente para convertirse en un ser superior ${ }^{38}$ más cerca de lo divino que de lo humano ${ }^{39}$.

La entrevista entre el Maestro y el discípulo concluye con la aceptación por parte de Paul Overt del consejo de Mr. St. George acerca de la renuncia que ha de conllevar la vocación artística entendida en su máxima pureza ${ }^{40}$. El discípulo le expresa su intención de llevar a cabo su objetivo: "I want to live in the greatest sense" (174), ante lo cual el Maestro muestra su admiración hacia él ${ }^{41}$. Este acontecimiento marca una inflexión en el desarrollo del relato puesto que a partir de este momento se produce una evidente inversión de funciones, más acentuada todavía con la muerte de la esposa de Mr. St. George.

En efecto, este funesto suceso (177) provoca la aparición de cierta contradicción en la hasta entonces congruente exposición doctrinal del Maestro. Paradójicamente, Mr. St. George dice por carta a su discípulo que su mujer había sido una influencia positiva para él, obviando toda referencia a cualquier tipo de discordia. Paul Overt deduce de estas palabras que las teorías de su admirado escritor en relación con el tema de la renuncia eran un error ${ }^{42}$. Eso proyecta la duda en el discípulo, aunque finalmente decide continuar (178) a fin de obtener la perfección ansiada ${ }^{43}$, cuya consecución constituirá no sólo la culminación de su carrera profesional, sino de algo que atañe directamente a su realización personal.

Tras conocer el compromiso entre Miss Fancourt y Mr. St. George, Paul Overt considera una mistificación las palabras de su Maestro (182), recordando la enseñanza recibida y tan disciplinadamente seguida (174).

32 "I'd turn myself inside down to save you" (165).

33 "Make people wriggle to another tune" (168).

34 "Conventional, material and brutalised life" (169).

35 "You don't know what we haven't got. It's bigger than all the rest" (169).

36 "He must be able to be poor" (173).

37 "Let him have all the passions he likes -if he only keeps his independence" (173).

38 "That's what makes them so superior" (172).

39 "He has only to do with the absolute; and a dear little family may represent a dozen relatives" (172-3). Obsérvese el juego de palabras entre absolute y relatives, que se centra en el equívoco del segundo término en su doble acepción: a) parientes y b) como antónimo de absolute.

40 "A life in which the passion -ours- is really intense" (174).

41 "Count on my highest appreciation, my devotion" (174).

42 "Renunciation was a mistake" (177).

43 "Something within him warned him he must make it supremely good" (178). 
La duda acerca de si su proceder ha valido la pena llega hasta el final (188), donde Henry James interviene con su comentario: "the Nature had dedicated him to intellectual, not to personal passion" (188), para expresar a modo de corolario que el sacrificio del verdadero artista es una especie de llamada a la que pocos están dispuestos a atender.

C) "The Death of the Lion":

El protagonista de "The Death of the Lion" presenta características semejantes a las del Doctor Hugh de "The Middle Years", sacrificando su trabajo como periodista por permanecer junto a su ídolo y cumplir la misión que considera debe llevar a cabo.

El relato evidencia la oposición entre las ideas de éxito social y la tarea del artista. En ese sentido, explora las relaciones entre el escritor y el lector desde un ángulo diferente al considerado en "The Middle Years". Allí el Doctor Hugh trataba de dar consistencia a la función del escritor, mientras que aquí, el protagonista intenta desvincular al artista del concepto de triunfo social, para restringir su campo de acción a un ámbito más intelectual y menos inmerso en la vida mundana.

Henry James comienza analizando la forma de trabajar de los medios de comunicación, y utiliza como protagonista-narrador a un periodista empleado en una empresa que acaba de cambiar de dueño. Él mismo se autodefine como perteneciente al "old lowering system" (261), seguidor de la escuela del anterior propietario. Frente a ello se presenta la figura del nuevo editor, Mr. Pinhorn, quien centra su línea periodística en crear al público sus necesidades y orientar su gusto ${ }^{44}$, para lo cual, uno de los instrumentos de los que se sirve será la mitificación de la figura del escritor a través de la prensa ${ }^{45}$, con un proceder que se basará en el instinto comercial puro y duro ${ }^{46}$. Y cuando la figura de Mr. Pinhorn se diluya, será la de Mr. Morrow quien tome el testigo de sus principios y el que encarnará el antagonista ideológico del narrador. Mr. Morrow, al igual que antes lo hiciera Mr. Pinhorn, se interesará fundamentalmente por los "surroundings" (271) y, además, pretenderá utilizar a un escritor ya consagrado para catapultar a otros que aspiran a abrirse camino en el difícil oficio de escritor, como es el caso de Dora Forbes ${ }^{47}$.

Pero de forma paradójica, Mr. Morrow influirá positivamente en nuestro protagonista, puesto que si bien le repulsan los métodos utilizados por su compañero -semejantes a los empleados por Mr. Pinhorn-, encuentra en ese odio hacia él la fuerza necesaria para romper con ese tipo de periodismo que le habían impuesto, consiguiendo llevar a cabo un cambio radical de comportamiento hacia el escritor objeto de su reportaje, Neil Paraday ${ }^{48}$ (271), lo que le induce a calificar de traición su actitud anterior, frente a la idea de salvación que guía ahora su nueva conducta:

I saw just how emphatic I should make my rejoinder to Mr Pinhorn, and that having come, like Mr Morrow, to betray, I must remain as long as possible to save. (272)

\footnotetext{
44 "Create the demand we required" (262).

45 "I see -you want to write him up" (262).

46 "He had smelt the coming glory as an animal smells its distant prey" (264).

${ }^{47}$ "But an authoritative word from Mr. Paraday -from the point of view of his sex, you know- would go right round the globe" (273).

${ }^{48}$ En realidad, ya anteriormente se habían producido los primeros síntomas de esta transformación: "I asked myself if I were the same young man who had come a few days before to scatter him to four winds" (269).
} 
E incluso considerará esta repentina metamorfosis como una auténtica conversión:

the little incident of my having gone down into the country for a profane purpose and been converted on the spot to holiness (286)

De ahora en adelante, su único propósito se orientará hacia la protección de la figura del escritor, intentando hacer que recupere "his old programme, his old ideal" (280), arruinado por el éxito que, no obstante, lo ha encumbrado a la consideración de "the king of the beasts of the year" (278). El prestigio de Neil Paraday es notorio: sin embargo, el aumento de su presencia en la vida social no tiene nada que ver con un interés propiamente literario ${ }^{49}$. Simultáneamente, surgirá otro protector de Neil Paraday, Mrs. Weeks Wimbush que competirá con nuestro protagonista en su cometido, pero desde un punto de vista más relacionado con el aspecto social que el propiamente artístico (280).

$\mathrm{Y}$ una vez expuestas las dos perspectivas que se contraponen a lo largo del relato, abordaremos la línea defendida por el protagonista, cuyo referente esencial lo constituye lo que él denomina el "study of my author's talent" (265), centrado en el valor artístico, radicalmente alejado del éxito comercial que aparece representado por el término "successful" (268). Así vemos que las condiciones apropiadas para la producción artística vienen determinadas de forma muy reveladora por medio de las siguientes expresiones: "the monastic life, the pious illumination of the missal in the convent-cell" (280). Y ese estudio minucioso de una obra literaria reviste una serie de características en estrecha relación con los conceptos de "revelations" y "perfection" (276), que son objeto de explicación por parte del narrador. Por una parte, las mencionadas "revelations" deben ser obtenidas a través de un proceso que requiere un esfuerzo intelectual por parte del lector: "extract the opinion, disengage the answer", "let us (...) interpret them [these pages]" (276). Y por otra, esa "perfection" hace referencia no sólo a la forma ${ }^{50}$, sino también al contenido ${ }^{51}$ de una obra. Pero ambos conceptos se unen para crear la idea de la existencia de un mensaje transcendental en la obra literaria que, si bien permanece oculto para la inmensa mayoría, puede ser descubierto por un restringido número de lectores. En este sentido, la frase " "no one has the faintest conception of what I'm trying for" "(278) trata de contrastar la escasa importancia concedida a la obra de un escritor famoso con el interés mostrado por compartir con él una vida social intensa: "“and not many have read three pages that I've written; but I must dine with them first (...)" "(278).

En suma, con "The Death of the Lion" Henry James sitúa el foco de atención en dos aspectos fundamentales, siendo el segundo consecuencia del primero:

a) Se presenta la vocación artística como un arduo sendero que exige una vida de dedicación máxima y exclusiva.

b) El fruto de ese sacrificio se ha de reflejar en forma de un mensaje de carácter esotérico que debe transmitir toda obra literaria de calidad, lo cual es objeto de detenido estudio en "The Figure in the Carpet".

D) "The Figure in the Carpet":

En este relato, Henry James establece diferentes niveles de comprensión de una obra literaria. Pero, sobre todo, lo que al autor le interesa resaltar es esa capacidad de una obra maestra para transmitir un mensaje codificado tan sólo comprensible para una élite. De ahí,

\footnotetext{
49 “"Not many have read three pages that I've written; but I must dine with them first (...)" (278).

${ }^{50}$ Expresada a través de las nociones de "tone" y "style" (276).

51 "The whole treatment of his case" (276).
}

Odisea, $n^{0} 1,2001$ 
las referencias varias a la intencionalidad profunda del texto artístico, que se fundamenta en los siguientes apartados:

A) Como justificación de la vocación artística, de la actividad del creador, del artista, del autor, para quien la intencionalidad de la obra es concebida como una pasión vital expuesta por medio de la frase "the very passion of the passion" (365) o también "the joy of my soul" (369).

B) Además habría una especie de comunicación entre el autor y el lector, en dos niveles:

a) Encuadrado dentro del ámbito fillosófico, como indican las frases: "some idea about life, some sort of philosophy, the organ of life" (368).

b) Y se llega incluso a entrar en el terreno de la iniciación esotérica, con expresiones tales como: "secret, esoteric message" (367). Se trata, en definitiva, de una búsqueda de la clave misteriosa -la denominada "figure in the carpet" (374, 381, 387, 392 y 394), expresión que da título al relato-, a través de la cual se puede acceder al "buried treasure" (384) compuesto de "gold and gems" (384).

C) En último extremo, nos encontramos con los destinatarios, en este caso los lectores, en donde se han de distinguir al menos tres grupos:

a) Primero, estaría el lector corriente, cuya percepción del significado es bastante precaria, sin conseguir captar el sentido profundo de la obra: "Nobody sees anything!"” (363).

b) Después, tenemos al crítico, quien constituiría un lector especializado, que consigue abrir ciertas vías de interpretación para el resto de los lectores: "the critics at least had put on a spurt and caught up with him. We had found out at last how clever he was" (360).

c) Y finalmente, vendría el auténtico iniciado, uno de los pocos que descubriría el significado último, escondido de forma magistral por el autor entre las líneas de su obra.

'(...) The order, the form, the texture of my books will perhaps some day constitute for the initiated a complete representation of it. So it's naturally the thing for the critic to look for (...)'. (366)

Así pues, el verdadero crítico literario debería poseer esa iniciación que le permitiera acceder al "buried treasure" (369) del conocimiento:

'But you talk about the initiated. There must therefore, you see, be initiation.'

'What else in heaven's name is criticism supposed to be?' (366)

Y hasta cierto punto, eso no constituye más que un reflejo de lo que Henry James pretendía que fuera su obra, al intentar hacer de ella un proceso activo en el que el lector debe interpretar el significado, a partir de las indicaciones dadas por el narrador ${ }^{52}$.

52 "El narrador de James está realmente implicado en la acción, pero separado de ella; tiene un papel en la historia, pero actúa principalmente como intermediario para el lector proporcionándole, no la interpretación completa de la escena, sino las claves para que él mismo pueda interpretarla." (Álvarez Calleja, 1988: 157).

Odisea, $n^{0} 1,2001$ 


\section{A MODO DE CONCLUSIÓN}

Ahora nos corresponde poner de relieve una serie de ideas que se desprenden del análisis de los cuatro relatos abordados en nuestro estudio, intentando vislumbrar las relaciones existentes con ciertos elementos del período socio-histórico e intelectual en el que se inscriben.

Ante todo, debemos destacar la identificación que se produce entre el artista y la función artística, alcanzando un grado tal que llega a relegar a un segundo plano la vida personal del escritor, despojándola de toda consistencia y relevancia:

el artista desaparecía dentro de su obra, la vida del escritor tenía interés tan sólo hasta el punto en que el arte se convertía en la única preocupación de su existencia y la propia personalidad ya no era interesante para tema de ficción. Como recomiendan muchas de sus historias sobre escritores -'The Figure in the Carpet', 'The Death of the Lion', 'The Lesson of the Master', etc.- la vida esencial del artista se encuentra en su obra, que es donde se afirma con mayor intensidad su genio característico, llegando ésta a convertirse en una especie de suprema autobiografía. (Álvarez Calleja, 1990: 207)

Se establece, por tanto, una especie de correlación conceptual implícita en la que la existencia corresponde a la vida personal del autor, limitada por las pequeñas contingencias materiales y subordinada en todo momento a su misión artística, que se erige así en la vía de escape hacia una sublimación de su ideal, convertido, de esta forma, en su esencia vital. Y en ello influyeron de forma decisiva los cambios introducidos por la Segunda Revolución Industrial en la sociedad de la segunda mitad del siglo XIX, con un ascenso de la burguesía como clase social, aupada gracias al enriquecimiento producido por el advenimiento de la economía capitalista. Ese mundo dominado por el dinero y el materialismo en el que Henry James se movía parecía ahogar las expectativas artísticas entendidas en su sentido estricto. Lo hemos visto de diversas maneras a lo largo de los relatos anteriormente considerados: el Doctor Hugh sacrifica su seguridad material -al abandonar a la Condesa- para estar junto a su admirado escritor; Neil Paraday siente cómo su producción literaria le ha servido para conseguir "éxito social" y convertirse en un personaje famoso, pero sus obras realmente no son leídas por quienes tanto lo alaban; Paul Overt renuncia a formar una familia para alcanzar la perfección artística y, en "The Figure in the Carpet", tanto George Corvick como su compañero hacen de la búsqueda de la clave oculta que subyace en los escritos de Hugh Vereker la finalidad casi exclusiva de sus vidas, llegando a ser para ellos una especie de piedra filosofal.

Esta percepción nos remite necesariamente al marco intelectual de fines del siglo XIX, en donde sobresale el pensamiento fecundo de Nietzsche, quien concibe el arte como la expresión más elevada del hombre (Nietzsche, 1980: 39). Y al igual que hemos visto en los relatos de Henry James, el filósofo alemán propugna la búsqueda de la perfección del ser por medio de la disciplina, el sacrificio y la renuncia, evitando todo lo que pueda apartarlo de su objetivo primordial $^{53}$ (Nietzsche, 1984: 128-30).

Para Nietzsche, además, el arte sirve de impulso al hombre para la suprema dignificación de su existencia (Nietzsche, 1980: 66), otorgándole un carácter metafísico (Nietzsche, 1980: 39) que presenta analogías no sólo con el tema de "The Middle Years" -e incluso con "The Figure in the Carpet"-, sino también con el Henry James de The Art of Fiction, cuando habla de

${ }^{53}$ Recuérdese en este sentido lo expuesto en "The Lesson of the Master" y "The Death of the Lion". 
esa capacidad del escritor para obtener de las "experiencias" de la vida una especie de "revelación", al pasar por el filtro de su "sensibilidad" artística:

Experience is never limited, and it is never complete; it is an immense sensibility (...). It is the very atmosphere of the mind; and when the mind is imaginative -much more when it happens to be that of a man of genius- (...) it converts the very pulses of the air into revelations. (James, 1985: 436)

La perfección de la obra artística adquiere en la mente de Henry James una dimensión gnoseológica -en línea claramente con esa "revelación" referida-, orientada a alcanzar los máximos niveles de conocimiento a través de las nociones de "beauty" y "truth":

In proportion as that intelligence is fine will the novel, the picture, the statue partake of the substance of beauty and truth. (James, 1985: 436)

Asimismo, se advierten algunas similitudes con los postulados preconizados por Stéphane Mallarmé, uno de los máximos exponentes del movimiento Simbolista francés, representado también por las figuras de Baudelaire, Rimbaud, Laforgue... Esas concomitancias con el poeta francés se aprecian en lo que se refiere a esa búsqueda de la esencia de las cosas frente a las apariencias contingentes, lo cual requiere una entrega total de uno mismo: la vocación artística exige una ascesis, una renuncia a los goces materiales e incluso un desprecio de la gloria (Lagarde y Michard, 1969: 529) bien patente tanto en "The Lesson of the Master" como en "The Death of the Lion". Para Mallarmé, la profesión del artista pasa a convertirse en un sacerdocio, impregnado de una capacidad de clarividencia que intenta destruir todas las barreras con el fin de desvelar el gran secreto (Lanson, 1951: 1123); de ahí que el arte sólo sea accesible -en último término- a unos pocos iniciados (Lanson, 1951: 1125), en clara sintonía con el argumento de "The Figure in the Carpet".

No hay duda de que los relatos en cuestión reflejan nítidamente los planteamientos mencionados, con lo cual podemos decir que las ideas de Henry James acerca de la función artística parecen anunciar la gestación del denominado Modernismo, fundamentalmente en lo que respecta al interés por el Arte como refugio frente al materialismo burgués -tan notorio en la sociedad americana de principios de siglo-, además de en lo concerniente a esa consideración de la imaginación artística como instrumento epistemológico alternativo al racionalismo realista (Cunliffe, 1993: 1-27; Ford, 1983: 281-353).

\section{BIBLIOGRAFÍA}

Álvarez Calleja, Ma. A. 1988. "El Teatro como elemento fundamental en la técnica narrativa de Henry James". Letras de Deusto, vol. XVIII, no 42: 145-57.

_ 1990. "El 'Prefacio Final' de Henry James”. Dianium: 201-8.

.1991. "Presencia de Henry James en la novelística del siglo XX". Studia Patriciae Shaw Oblata, vol. I, Oviedo: Servicio de Publicaciones de la Universidad de Oviedo. 3-11.

1994. "La novela experimental de Henry James: Dimensión poética de 'The Beast in the Jungle"”. Epos, vol. X: 415-30. 
Anderson, Ch. R. 1977. Person, Place and Thing in Henry James's Novels. Durham: Duke University Press.

Auchard, J. 1986. Silence in Henry James: The Heritage of Symbolism and Decadence. University Park and London: The Pennsylvania State University Press.

Cunliffe, M. 1991. The Literature of the United States. Harmondsworth: Penguin Books.

_ ., ed. 1993. The Penguin History of American Literature. American Literature since 1900, vol. IX. Harmondsworth: Penguin Books.

Ford, B., ed. 1983. The New Pelican Guide to English Literature. From James to Eliot, vol. 7. Harmondsworth: Penguin Books.

James, H. 1983. Autobiography. Princeton: Princeton University Press.

_ 1985. "The Art of Fiction". The Norton Anthology of American Literature, vol. II. New York - London: W. W. Norton \& Company.

. 1986. The Figure in the Carpet and Other Stories. Harmondsworth: Penguin Classics.

Lagarde, A. y Michard, L. 1969. XIXe. Siècle. Les Grands Auteurs Français. Paris: Bordas.

Lanson, G. 1951. Histoire de la Littérature Française (remaniée et completée pour la période 1850-1950 par Paul Tuffrau). Paris: Hachette.

Nietzsche, F. 1980. El Nacimiento de la Tragedia. Madrid: Alianza Editorial.

— 1984. La Genealogía de la Moral. Madrid: Alianza Editorial.

Perosa, S. 1978. Henry James and the Experimental Novel. Charlottesville: Virginia University Press. 